\title{
How to choose the right vaginal moisturizer or lubricant for your patient
}

\author{
About $20 \%$ of women report having used a vaginal lubrication product in \\ the past 30 days. Knowing what is in these widely used products can help \\ your patients select from the litany of options.
}

Jon F. Pennycuff, MD, MSPH, and Cheryl Iglesia, MD

V aginal dryness, encompassed in the modern term genitourinary syndrome of menopause (GSM) affects up to $40 \%$ of menopausal women and up to $60 \%$ of postmenopausal breast cancer survivors. ${ }^{1,2}$ Premenopausal women also can have vulvovaginal dryness while breastfeeding (lactational amenorrhea) and while taking low-dose contraceptives. ${ }^{3}$ Vaginal moisturizers and lubricants are the first-line treatment options for vaginal dryness, dyspareunia, and GSM. ${ }^{4,5}$ In fact, approximately two-thirds of women have reported using a vaginal lubricant in their lifetime. ${ }^{6}$ Despite such ubiquitous use, many health care providers and patients have questions about the difference

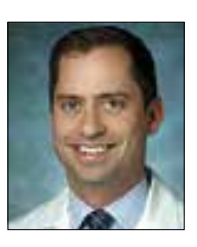

Dr. Pennycuff is Fellow, Female Pelvic Medicine and Reconstructive Surgery, Department of Obstetrics and Gynecology, MedStar Health, Washington DC.

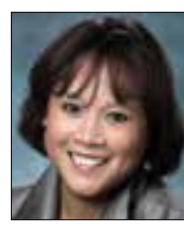

Dr. Iglesia is Section Director, Female Pelvic Medicine and Reconstructive Surgery, MedStar Washington Hospital Center; Associate Professor, Obstetrics, Gynecology and Urology, Georgetown University; and Assistant Professor, Department of Obstetrics and Gynecology, Uniformed Services University of the Health and Sciences, Bethesda, MD.

The authors report no financial relationships relevant to this article.

doi: 10.12788/obgm.0104 between vaginal moisturizers and lubricants and how to best choose a product.

\section{Vaginal moisturizers}

Vaginal moisturizers are designed to rehydrate the vaginal epithelium. Much like facial or skin moisturizers, they are intended to be applied regularly, every 2 to 3 days, but may be applied more often depending on the severity of symptoms. Vaginal moisturizers work by increasing the fluid content of the vaginal tissue and by lowering the vaginal $\mathrm{pH}$ to mimic that of natural vaginal secretions. Vaginal moisturizers are typically water based and use polymers to hydrate tissues. ${ }^{7}$ They change cell morphology but do not change vaginal maturation, indicating that they bring water to the tissue but do not shift the balance between superficial and basal cells and do not increase vaginal epithelial thickness as seen with vaginal estrogen. ${ }^{8}$ Vaginal moisturizers also have been found to be a safe alternative to vaginal estrogen therapy and may improve markers of vaginal health, including vaginal moisture, vaginal fluid volume, vaginal elasticity, and premenopausal $\mathrm{pH} .{ }^{9}$ Commercially available vaginal moisturizers have been shown to be as effective as vaginal estrogens in reducing vaginal symptoms such as itching, irritation, and dyspareunia, but some caution should be taken when interpreting these results as neither vaginal moisturizer nor vaginal estrogen tablet were more effective than placebo in a recent randomized controlled trial. ${ }^{10,11}$ Small
IN THIS ARTICLE

The effects of additives page 46

Available nonhormonal moisturizers

page 47

Common patient concerns page 49 
FAST

TRACK

Silicone-based

lubricants are

readily

available and

compatible with

latex condoms

and generally

require a smaller

amount than

water-based

lubricants studies on hyaluronic acid have shown efficacy for the treatment of vaginal dryness. ${ }^{12,13}$ Hyaluronic acid is commercially available as a vaginal suppository ovule and as a liquid. It may also be obtained from a reliable compounding pharmacy. Vaginal suppository ovules may be a preferable formulation for women who find the liquids messy or cumbersome to apply.

\section{Lubricants}

Lubricants differ from vaginal moisturizers because they are specifically designed to be used during intercourse to provide shortterm relief from vaginal dryness. They may be water-, silicone-, mineral oil-, or plant oilbased. The use of water- and silicone-based lubricants is associated with high satisfaction for intercourse as well as masturbation. ${ }^{14}$ These products may be particularly beneficial to women whose chief complaint is dyspareunia. In fact, women with dyspareunia report more lubricant use than women without dyspareunia, and the most common reason for lubricant use among these women was to reduce or alleviate pain..$^{15}$ Overall, women both with and without dyspareunia have a positive perception regarding lubricant use and prefer sexual intercourse that feels more "wet," and women in their forties have the most positive perception about lubricant use at the time of intercourse compared with other age groups. ${ }^{16}$ Furthermore, the World Health Organization (WHO) recommends that condom-compatible lubricants be used with condoms for menopausal and postmenopausal women. ${ }^{17}$ Both water-based and silicone-based lubricants may be used with latex condoms, while oil-based lubricants should be avoided as they can degrade the latex condom. While vaginal moisturizers and lubricants technically differ based on use, patients may use one product for both purposes, and some products are marketed as both a moisturizer and lubricant.

\section{Providing counsel to patients}

Patients often seek advice on how to choose vaginal moisturizers and lubricants. Understanding the compositions of these products and their scientific evidence is useful when helping patients make informed decisions regarding their pelvic health. Most commercially available lubricants are either wateror silicone- based. In one study comparing these two types of lubricants, water-based lubricants were associated with fewer genital symptoms than silicone-based products. ${ }^{14}$ Women may want to use a natural or organic product and may prefer plant-based oils such as coconut oil or olive oil. Patients should be counseled that latex condoms are not compatible with petroleum-, mineral oil- or plant oil-based lubricants.

In our practice, we generally recommend silicone-based lubricants, as they are readily available and compatible with latex condoms and generally require a smaller amount than water-based lubricants. They tend to be more expensive than water-based lubricants. For vaginal moisturizers, we often recommend commercially available formulations that can be purchased at local pharmacies or drug stores. However, a patient may need to try different lubricants and moisturizers in order to find a preferred product. We have included in TABLES 1 AND $2^{7,17,18}$ a list of commercially available vaginal moisturizers and lubricants with ingredient list, $\mathrm{pH}$, osmolality, common formulation, and cost when available, which has been compiled from $\mathrm{WHO}$ and published research data to help guide patient counseling. (Table 2 is available in the online version of this article at mdedge.com/obgyn.)

\section{The effects of additives}

Water-based moisturizers and lubricants may contain many ingredients, such as glycerols, fragrance, flavors, sweeteners, warming or cooling agents, buffering solutions, parabens and other preservatives, and numbing agents. These substances are added to water-based products to prolong water content, alter viscosity, alter $\mathrm{pH}$, achieve certain sensations, and prevent bacterial contamination. ${ }^{7}$ The addition of these substances, however, will alter osmolality and $\mathrm{pH}$ balance of 
TABLE 1 Commonly available nonhormonal vaginal moisturizers formulations

\begin{tabular}{|c|c|c|c|c|c|}
\hline Product & Ingredients & $\mathrm{pH}^{7,18}$ & $\begin{array}{c}\text { Osmolality } \\
(\mathrm{mOSm} / \mathrm{kg})^{7,18}\end{array}$ & $\begin{array}{c}\text { Formulation and } \\
\text { use }\end{array}$ & $\begin{array}{l}\text { Approximate } \\
\text { price }\end{array}$ \\
\hline RepHresh Vaginal gel & $\begin{array}{l}\text { Purified water USP, } \\
\text { glycerin, polycarbophil, } \\
\text { carbopol 974P, } \\
\text { ethylparaben sodium, } \\
\text { methylparaben sodium, } \\
\text { propylparaben sodium, } \\
\text { sodium hydroxide }\end{array}$ & 3.4 & $1,439-1,914$ & $\begin{array}{l}\text { Prefilled } \\
\text { applicator used } \\
\text { every } 3 \text { days }\end{array}$ & $\begin{array}{l}4 \text { applicators } \\
\text { (12 days) } \\
\$ 26.49\end{array}$ \\
\hline $\begin{array}{l}\text { Replens Long-Lasting } \\
\text { Vaginal Moisturizer }\end{array}$ & $\begin{array}{l}\text { Purified water, glycerin, } \\
\text { mineral oil, polycarbophil, } \\
\text { carbomer homopolymer } \\
\text { type B, hydrogenated } \\
\text { palm oil glyceride, sorbic } \\
\text { acid, sodium hydroxide }\end{array}$ & $2.95-3.0$ & $1,177-2,011$ & $\begin{array}{l}\text { Prefilled } \\
\text { applicator used } \\
\text { every } 3 \text { days }\end{array}$ & $\begin{array}{l}8 \text { applicators } \\
\text { (1-month supply) } \\
\$ 22.49\end{array}$ \\
\hline Luvena & $\begin{array}{l}\text { Water, propanediol, PEG- } \\
20, \text { xanthan gum, PEG- } \\
20 \mathrm{M} \text {, simmonsia } \\
\text { chinensis (Jojoba) } \\
\text { seed oil, vaccinium } \\
\text { macrocopon } \\
\text { (cranberry) fruit extract, } \\
\text { lysozyme, lactoferrin, } \\
\text { lactoperoxidase, } \\
\text { lactic acid, potassium } \\
\text { thiocynanate, glycogen, } \\
\text { mannose, tocopherol } \\
\text { (vitamin E) }\end{array}$ & Unavailable & Unavailable & $\begin{array}{l}\text { Prefilled } \\
\text { applicators, use } \\
\text { every } 3 \text { days as } \\
\text { moisturizer or } \\
\text { lubricant }\end{array}$ & $\begin{array}{l}6 \text { applicators for } \\
\$ 19.99\end{array}$ \\
\hline $\begin{array}{l}\text { Canesintima Intimate } \\
\text { Moisturizer }\end{array}$ & $\begin{array}{l}\text { Aqua, glycerin, glyceryl } \\
\text { polymethacrylate, cap- } \\
\text { ryloyl glycine, sorbitol, } \\
\text { acrylates/C10-30 alkyl } \\
\text { acrylate crosspolymer, } \\
\text { sodium hyaluronate, so- } \\
\text { dium benzoate, sodium } \\
\text { hydroxide, galactoara- } \\
\text { binan, butylene glycol/ } \\
\text { camellia japonica leaf/ } \\
\text { flower extract, tetrasodi- } \\
\text { um EDTA, P-anisic acid, } \\
\text { levulinic acid }\end{array}$ & 5.63 & 846 & Pump bottle & $\begin{array}{l}50 \mathrm{~mL} \text { pump } \\
\text { bottle for } \$ 33.49\end{array}$ \\
\hline $\begin{array}{l}\text { Ah! Yes Vaginal } \\
\text { Moisturizer }\end{array}$ & $\begin{array}{l}\text { Aqua (water), linum } \\
\text { usitatissimum (flax) } \\
\text { extract, aloe barbadensis } \\
\text { leaf juice powder (aloe } \\
\text { vera), ceratonia siliqua } \\
\text { (locust bean gum), cy- } \\
\text { amopsis tetragonolo- } \\
\text { bus (guar gum), sodium } \\
\text { chloride, xanthan gum, } \\
\text { potassium sorbate, citric } \\
\text { acid, phenoxyethanol }\end{array}$ & $3.8-4.2$ & $260-290$ & $\begin{array}{l}\text { Flip top bottle } \\
\text { (100 mL); prefilled } \\
\text { applicators used } \\
\text { every } 3 \text { days }\end{array}$ & $\begin{array}{l}\$ 14.99 \text { for flip top } \\
\text { bottle, } \$ 53.89 \text { for } \\
30 \text { applicators }\end{array}$ \\
\hline
\end{tabular}


TABLE 1 Commonly available nonhormonal vaginal moisturizers formulations (continued)

\begin{tabular}{|c|c|c|c|c|c|}
\hline Product & Ingredients & $\mathrm{pH}^{7,18}$ & $\begin{array}{c}\text { Osmolality } \\
(\mathrm{mOSm} / \mathrm{kg})^{7,18}\end{array}$ & $\begin{array}{l}\text { Formulation and } \\
\text { use }\end{array}$ & $\begin{array}{c}\text { Approximate } \\
\text { price }\end{array}$ \\
\hline $\begin{array}{l}\text { Sylk Natural Intimate } \\
\text { Moisturizer }\end{array}$ & $\begin{array}{l}\text { Purified water, kiwifruit } \\
\text { plant extract, citrus seed } \\
\text { extract, xanthan plant } \\
\text { extract, vegetable glycer- } \\
\text { ine, citric acid, potassium } \\
\text { sorbate, sodium citrate }\end{array}$ & 4.47 & 877 & $\begin{array}{l}3 \text { fluid oz flip top } \\
\text { tube }\end{array}$ & $\$ 22.95$ \\
\hline $\begin{array}{l}\text { HyaloGyn Vaginal } \\
\text { Hydrating Gel }\end{array}$ & $\begin{array}{l}\text { Hydeal-D® (hyaluronic } \\
\text { acid derivative), propyl- } \\
\text { ene glycol, carbomer } \\
\text { (carbopol 974P), methyl } \\
\text { p-hydroxybenzoate, pro- } \\
\text { pyl p-hydroxybenzoate, } \\
\text { sodium hydroxide, puri- } \\
\text { fied water }\end{array}$ & 4.88 & $1,336-1,729$ & $\begin{array}{l}30 \mathrm{~g} \text { tube with } 10 \\
\text { applicators } \\
(30 \text {-day supply) }\end{array}$ & $\$ 27.00$ \\
\hline $\begin{array}{l}\text { Revaree Vaginal } \\
\text { Moisturizer }\end{array}$ & $\begin{array}{l}\text { 2-g vaginal insert } \\
\text { contains } 5 \text { mg of hyal- } \\
\text { uronic acid sodium salt } \\
\text { in a base consisting of a } \\
\text { mixture of semi-synthetic } \\
\text { glycerides, which help } \\
\text { it retain its shape. (Full } \\
\text { ingredient list not listed.) }\end{array}$ & Unavailable & Unavailable & $\begin{array}{l}10 \text { vaginal } \\
\text { suppositories } \\
(30 \text {-day supply) }\end{array}$ & $\$ 55.00$ \\
\hline $\begin{array}{l}\text { K-Y Liquibeads Vaginal } \\
\text { Moisturizer }\end{array}$ & $\begin{array}{l}\text { Dimethicone, dimethi- } \\
\text { conol, gelatin, glycerin }\end{array}$ & Unavailable & Unavailable & 6 vaginal ovules & $\$ 22.79$ \\
\hline
\end{tabular}

the product, which may be of clinical consequence. Silicone- or oil-based products do not contain water and therefore do not have a $\mathrm{pH}$ or an osmolality value.

Hyperosmolar formulations can theoretically injure epithelial tissue. In vitro studies have shown that hyperosmotic vaginal products can induce mild to moderate irritation, while very hyperosmolar formulations can induce severe irritation and tissue damage to vaginal epithelial and cervical cells. ${ }^{19,20}$ The WHO recommends that the osmolality of a vaginal product not exceed $380 \mathrm{mOsm} / \mathrm{kg}$, but very few commercially available products meet these criteria so, clinically, the threshold is $1,200 \mathrm{mOsm} / \mathrm{kg} .{ }^{17}$ It should be noted that most commercially available products exceed the $1,200 \mathrm{mOsm} / \mathrm{kg}$ threshold. Vaginal products may be a cause for vaginal irritation and should be considered in the differential diagnosis.

The normal vaginal $\mathrm{pH}$ is $3.8-4.5$, and vaginal products should be $\mathrm{pH}$ balanced to this range. The exact role of $\mathrm{pH}$ in these products remains poorly understood. Nonetheless, products with a $\mathrm{pH}$ of 3 or lower are not recommended..$^{18}$ Concerns about osmolality and $\mathrm{pH}$ remain theoretical, as a study of 12 commercially available lubricants of varying osmolality and $\mathrm{pH}$ found no cytotoxic effect in vivo. ${ }^{18}$

Vaginal moisturizers and lubricants contain many inactive ingredients, the most controversial of which are parabens. These substances are used in many cosmetic products as preservatives and are weakly estrogenic. These substances have been found in breast cancer tissue, but their possible role as a carcinogen remains uncertain..$^{21,22}$ Nonetheless, the use of paraben-containing products is not recommended for women who have a history of hormonally-driven cancer or who are at high risk for developing cancer. ${ }^{7}$ Many lubricants contain glycerols (glycerol, glycerine, and propylene glycol) to alter viscosity or alter the water properties. The WHO recommends limits on the content of glycerols in these products. ${ }^{17}$ Glycerols have been 
associated with increased risk of bacterial vaginosis (adjusted odds ratio [aOR], 11.75; 95\% confidence interval [CI], 1.96-70.27), and can serve as a food source for candida species, possibly increasing risk of yeast infections. $^{7,23}$ Additionally, vaginal moisturizers and lubricants may contain preservatives such as chlorhexidine, which can disrupt normal vaginal flora and may cause tissue irritation. ${ }^{7}$

\section{Common concerns to be aware of}

Women using vaginal products may be concerned about adverse effects, such as worsening vaginal irritation or infection. Vaginal moisturizers have not been shown to have increased risk of adverse effects compared with vaginal estrogens. ${ }^{9,10}$ In vitro studies have shown that vaginal moisturizers and lubricants inhibit the growth of Escherichia coli but may also inhibit Lactobacillus crispatus. ${ }^{24}$ Clinically, vaginal moisturizers have been shown to improve signs of bacterial vaginosis and have even been used to treat bacterial vaginosis. ${ }^{25,26}$ A study of commercially available vaginal lubricants inhibited the growth

\section{References}

1. Castelo-Branco C, Cancelo MJ, Villero J, et al. Management of postmenopausal vaginal atrophy and atrophic vaginitis. Maturitas. 2005;52(suppl 1):S46-S52. doi: 10.1016/j. maturitas.2005.06.014

2. Crandall C, Peterson L, Ganz PA, et al. Association of breast cancer and its therapy with menopause-related symptoms. Menopause. 2004;11:519-530. doi: 10.1097/01. gme.0000117061.40493.ab

3. Bornstein J, Goldstein AT, Stockdale CK, et al. 2015 ISSVD, ISSWSH, and IPPS Consensus Terminology and Classification of Persistant Vulvar Pain and Vulvodynia. J Sex Med. 2016;13:607-612. doi: 10.1016/j.jsxm.2016.02.167.

4. American College of Obstetricians and Gynecologists. ACOG Practice Bulletin No. 141: management of menopausal symptoms. Obstet Gynecol. 2014;123:202-216. doi: 10.1097/01.AOG.0000441353.20693.78.

5. Faubion S, Larkin L, Stuenkel C, et al. Management of genitourinary syndrome of menopause in women with or at high risk for breast cancer: consensus recommendation from The North American Menopause Society and the International Society for the Study for Women's Sexual Health. Menopause. 2018;25:596-608. doi: 10.1097 /GME.0000000000001121.

6. Herbenick D, Reece M, Schick V, et al. Women's use and perceptions of commercial lubricants: prevalence and characteristics in a nationally representative sample of American adults. J Sex Med. 2014;11:642-652. doi: 10.1111 /jsm.12427.

7. Edwards D, Panay N. Treating vulvovaginal atrophy/ genitourinary syndrome of menopause: how important is vaginal lubricant and moisturizer composition? Climacteric. 2016;19:151-116. doi: 10.3109/13697137.2015.1124259. of $L$ crispatus, which may predispose to irritation and infection. ${ }^{27}$ Nonetheless, the effect of the vaginal products on the vaginal microbiome and vaginal tissue remains poorly studied. Vaginal moisturizers and lubricants, while often helpful for patients, also can potentially cause irritation or predispose to infections. Providers should consider this when evaluating patients for new onset vaginal symptoms after starting vaginal products.

\section{Bottom line}

Vaginal products such as moisturizers and lubricants are often effective treatment options for women suffering from genitourinary syndrome of menopause and may be first-line treatment options, especially for women who may wish to avoid estrogen-containing products. Vaginal moisturizers can be recommended to any women experiencing vaginal irritation due to vaginal dryness while vaginal lubricants should be recommended to sexually active women who experience dyspareunia. Clinicians need to be aware of the formulations of these products and possible side effects in order to appropriately counsel patients.

8. Van der Lakk JAWN, de Bie LMT, de Leeuw H, et al. The effect of Replens on vaginal cytology in the treatment of postmenopausal atrophy: cytomorphology versus computerized cytometry. J Clin Pathol. 2002;55:446-451. doi: 10.1136/jcp.55.6.446.

9. Nachtigall LE. Comparitive study: Replens versus local estrogen in menopausal women. Fertil Steril. 1994;61:178180. doi: 10.1016/s0015-0282(16)56474-7.

10. Bygdeman M, Swahn ML. Replens versus dienoestrol cream in the symptomatic treatment of vaginal atrophy in postmenopausal women. Maturitas. 1996;23:259-263. doi: 10.1016/0378-5122(95)00955-8.

11. Mitchell CM, Reed SD, Diem S, et al. Efficacy of vaginal estradiol or vaginal moisturizer vs placebo for treating postmenopausal vulvovaginal symptoms. JAMA Intern Med. 2018;178:681-690. doi: 10.1001/jamainternmed.2018.0116.

12. Chen J, Geng L, Song X, et al. Evaluation of the efficacy and safety of hyaluronic acid vaginal gel to ease vaginal dryness: a multicenter, randomized, controlled, open-label, parallelgroup, clinical trial. J Sex Med. 2013;10:1575-1584. doi: $10.1111 /$ jsm.12125.

13. Jokar A, Davari T, Asadi N, et al. Comparison of the hyaluronic acid vaginal cream and conjugated estrogen used in treatment of vaginal atrophy of menopause women: a randomized controlled clinical trial. IJCBNM. 2016;4:69-78.

14. Herbenick D, Reece M, Hensel D, et al. Association of lubricant use with women's sexual pleasure, sexual satisfaction, and genital symptoms: a prospective daily diary study. J Sex Med. 2011;8:202-212. doi: 10.1111/j.17436109.2010.02067.x.

15. Sutton KS, Boyer SC, Goldfinger C, et al. To lube or not to lube: experiences and perceptions of lubricant use in women 


\section{Choosing the right vaginal lubricant or moisturizer for your patient}

with and without dyspareunia. J Sex Med. 2012;9:240-250. doi: 10.1111/j.1743-6109.2011.02543.x.

16. Jozkowski KN, Herbenick D, Schick V, et al. Women's perceptions about lubricant use and vaginal wetness during sexual activity. J Sex Med. 2013;10:484-492. doi: 10.1111 /jsm.12022.

17. World Health Organization. Use and procurement of additional lubricants for male and female condoms: WHO /UNFPA/FHI360 advisory note. 2012. https://www.who. int/reproductivehealth/publications/rtis/rhr12_33/en/. Accessed February 13, 2021

18. Cunha AR, Machado RM, Palmeira de Oliveira A, et al. Characterization of commercially available vaginal lubricants: a safety perspective. Pharmaceuticals. 2014;6:530542. doi: 10.3390/pharmaceutics6030530.

19. Adriaens E, Remon JP. Mucosal irritation potential of personal lubricants relates to product osmolality as detected by the slug mucosal irritation assay. Sex Transm Dis. 2008;35:512516. doi: 10.1097/OLQ.0b013e3181644669.

20. Dezzuti CS, Brown ER, Moncla B, et al. Is wetter better? An evaluation of over-the-counter personal lubricants for safety and anti-HIV activity. PLoS One. 2012;7:e48328. doi: 10.1371 /journal.pone.0048328.

21. Harvey PW, Everett DJ. Significance of the detection of esters of p-hydroxybenzoic acid (parabens) in human breast tumours. J Appl Toxicol. 2004:24:1-4. doi: 10.1002/jat.957.

22. Darbre PD, Alijarrah A, Miller WR, et al. Concentrations of parabens in human breast tumous. J Appl Toxicol. 2004;24:513. doi: $10.1002 /$ jat.958.

23. Brotman RM, Ravel J, Cone RA, et al. Rapid fluctuation of the vaginal microbiota measured by Gram stain analysis. Sex Transm Infect. 2010;86:297-302. doi: 10.1136/sti.2009.040592.

24. Hung KJ, Hudson P, Bergerat A, et al. Effect of commercial vaginal products on the growth of uropathogenic and commensal vaginal bacteria. Sci Rep. 2020;10:7625.

25. Wu JP, Fielding SL, Fiscell K. The effect of the polycarbophil gel (Replens) on bacterial vaginosis: a pilot study. Eur J Obstet Gynecol Reprod Biol. 2007;130:132-136. doi: 10.1016/j. ejogrb.2006.01.007.

26. Fiorelli A, Molteni B, Milani M. Successful treatment of bacterial vaginosis with a polycarbophil-carbopol acidic vaginal gel: results from a randomized double-bling, placebo controlled trial. Eur J Obstet Gynecol Reprod Biol. 2005;120:202-205. doi: 10.1016/j.ejogrb.2004.10.011.

27. Fashemi B, Delaney ML, Onderdonk AB, et al. Effects of feminine hygiene products on the vaginal mucosal biome. Microb Ecol Health Dis. 2013;24. doi: 10.3402/mehd. v24i0.19703. 
TABLE 2 Commonly available nonhormonal lubricants

\begin{tabular}{|c|c|c|c|c|c|}
\hline Product & Ingredients & $\mathrm{pH}^{7,17,18}$ & $\begin{array}{c}\text { Osmolality } \\
(\mathrm{mOSm} / \mathrm{kg})^{7,17,18}\end{array}$ & $\begin{array}{c}\text { Formulation } \\
\text { and use }\end{array}$ & $\begin{array}{c}\text { Approximate } \\
\text { price }\end{array}$ \\
\hline $\begin{array}{l}\text { K-Y Jelly } \\
\text { Water Based } \\
\text { Lubricant }\end{array}$ & $\begin{array}{l}\text { Water, glycerin, } \\
\text { hydroxyethylcellulose, } \\
\text { chlorhexidine, gluconate, } \\
\text { gluconolactone, methylparaben, } \\
\text { sodium hydroxide }\end{array}$ & $3.5-4.49$ & $2,007-3,631$ & $\begin{array}{l}2 \text { and } 4 \text { oz flip } \\
\text { top bottle }\end{array}$ & $\$ 4.99-\$ 7.59$ \\
\hline $\begin{array}{l}\text { Astroglide Gel } \\
\text { Lubricant }\end{array}$ & $\begin{array}{l}\text { Purified water, glycerin, } \\
\text { hydroxyethylcellulose, chlorhexidine } \\
\text { gluconate, methylparaben, glucono } \\
\text { delta lactone, sodium hydroxide }\end{array}$ & 4.38 & 6,100 & 4 oz flip top tube & $\$ 6.79$ \\
\hline $\begin{array}{l}\text { ID Glide } \\
\text { Lubricant }\end{array}$ & $\begin{array}{l}\text { Water, glycerin, propylene glycol, } \\
\text { cellulose gum, EDTA, carbomer, } \\
\text { PEG-90M, tetrahydroxypropyl } \\
\text { ethylenediamine, methylparaben, } \\
\text { sodium benzoate, potassium } \\
\text { sorbate }\end{array}$ & $5.20^{a}$ & $3,200^{a}$ & Multiple & $\$ 3.49-159.99$ \\
\hline $\begin{array}{l}\text { Wet Original } \\
\text { Gel Lubricant }\end{array}$ & $\begin{array}{l}\text { Water (aqua), glycerin, } \\
\text { carboxymethylcellulose, pentylene } \\
\text { glycol, potassium sorbate }\end{array}$ & 5.9 & 3,679 & $5-32$ oz bottles & $\$ 8.33-\$ 39.51$ \\
\hline $\begin{array}{l}\text { Durex Play Feel } \\
\text { Pleasure Gel }\end{array}$ & $\begin{array}{l}\text { Purified water, propylene glycol, } \\
\text { hydroxyethylcellulose, benzoic acid, } \\
\text { sodium hydroxide }\end{array}$ & 5.48 & 1,563 & $\begin{array}{l}50 \mathrm{~mL} \text { flip top } \\
\text { bottle }\end{array}$ & $\$ 8.99$ \\
\hline $\begin{array}{l}\text { Good Clean } \\
\text { Love Almost } \\
\text { Naked Organic } \\
\text { Personal } \\
\text { Lubricant } \\
\end{array}$ & $\begin{array}{l}\text { Organic aloe barbadensis leaf } \\
\text { juice, xanthan gum, agar, lactic } \\
\text { acid, potassium sorbate, sodium } \\
\text { benzoate, natural flavor }\end{array}$ & 4.73 & 240 & 4 oz flip top tube & $\$ 11.99$ \\
\hline $\begin{array}{l}\text { H2O Sliquid } \\
\text { Naturals }\end{array}$ & $\begin{array}{l}\text { Purified water, plant cellulose } \\
\text { (from cotton), cyamopsis (guar } \\
\text { conditioners), potassium sorbate, } \\
\text { citric acid }\end{array}$ & $4.0-4.4$ & Unavailable & $\begin{array}{l}4.2 \text { or } 8.5 \mathrm{oz} \\
\text { bottles }\end{array}$ & $\$ 12.00-\$ 19.00$ \\
\hline $\begin{array}{l}\text { Pjur Med } \\
\text { Natural Glide } \\
\text { Personal } \\
\text { Lubricant } \\
\end{array}$ & $\begin{array}{l}\text { Aqua (water), glycerin, xanthan gum, } \\
\text { benzyl alcohol, sodium benzoate, } \\
\text { potassium sorbate, citric acid }\end{array}$ & 4.41 & $>2,000$ & $100 \mathrm{~mL}$ bottle & $\$ 15.95$ \\
\hline $\begin{array}{l}\text { Pjur Women } \\
\text { Nude Lubricant }\end{array}$ & $\begin{array}{l}\text { Aqua (water), propylene glycol, } \\
\text { ethoxydiglycol, hydroxypropyl guar } \\
\text { hydroxypropyltrimonium chloride, } \\
\text { hydroxyethyl cellulose, sodium } \\
\text { saccharin, citric acid }\end{array}$ & 4.42 & $>2,000$ & $100 \mathrm{~mL}$ bottle & $\$ 34.99$ \\
\hline $\begin{array}{l}\text { Ritex Senstiv } \\
\text { Gel }\end{array}$ & $\begin{array}{l}\text { Aqua, glycerin, butylene glycol, } \\
\text { hydroxyethylcellulose, sodium } \\
\text { lactate, lactic acid }\end{array}$ & 4.04 & $>2,000$ & $100 \mathrm{~mL}$ tube & $\$ 48.99$ \\
\hline $\begin{array}{l}\text { Simply Slick } \\
\text { Personal } \\
\text { Lubricating } \\
\text { Lotion }\end{array}$ & $\begin{array}{l}\text { Castor oil, purified water, jojoba oil, } \\
\text { vegetable glycerin, pectin, Stevia, } \\
\text { Optiflo H370VF }\end{array}$ & 6.68 & $>2,000$ & 2 oz bottle & $\$ 17.77$ \\
\hline
\end{tabular}


TABLE 2 Commonly available nonhormonal lubricants (continued)

\begin{tabular}{|c|c|c|c|c|c|}
\hline Product & Ingredients & $\mathrm{pH}^{7,17,18}$ & $\begin{array}{c}\text { Osmolality } \\
(\mathrm{mOSm} / \mathrm{kg})^{7,17,18}\end{array}$ & $\begin{array}{l}\text { Formulation } \\
\text { and use }\end{array}$ & $\begin{array}{l}\text { Approximate } \\
\text { price }\end{array}$ \\
\hline $\begin{array}{l}\text { Ah! Yes } \\
\text { Water Based } \\
\text { Lubricant }\end{array}$ & $\begin{array}{l}\text { Aqua (water), aloe barbadensis } \\
\text { leaf juice powder (aloe vera), linum } \\
\text { usitatissimum (flax) seed extract, } \\
\text { cyamopsis tetragonolobus (guar } \\
\text { gum), ceratonia siliqua (locust } \\
\text { bean gum), xanthan gum, sodium } \\
\text { chloride, potassium sorbate, citric } \\
\text { acid, phenoxyethanol }\end{array}$ & 4.08 & 154 & $\begin{array}{l}1.7-5.1 \text { oz flip } \\
\text { top tubes }\end{array}$ & $\$ 8.49-\$ 21.99$ \\
\hline Uber Lube & $\begin{array}{l}\text { Dimethicone, dimethiconol, } \\
\text { cyclomethicone, tocopheryl acetate } \\
\text { (vitamin E) }\end{array}$ & 4.6 & $\mathrm{~N} / \mathrm{A}$ & $\begin{array}{l}50 \text { and } 100 \mathrm{~mL} \\
\text { pump glass } \\
\text { bottle }\end{array}$ & $\$ 18.00-\$ 28.00$ \\
\hline $\begin{array}{l}\text { Swiss Navy } \\
\text { Silicone } \\
\text { Lubricant }\end{array}$ & $\begin{array}{l}\text { Cyclopentasiloxane, dimethicone, } \\
\text { tocopheryl acetate }\end{array}$ & N/A & $\mathrm{N} / \mathrm{A}$ & 4 oz pump bottle & $\$ 16.49$ \\
\hline $\begin{array}{l}\text { K-Y True Feel } \\
\text { Premium } \\
\text { Silicone } \\
\text { Lubricant }\end{array}$ & Dimethicone & N/A & N/A & $1.5 \mathrm{oz}$ bottle & $\$ 13.99$ \\
\hline
\end{tabular}

aFormulation of product changed since laboratory testing so $\mathrm{pH}$ and osmolality may be different. 